\title{
Gold(I)-Catalyzed Ring Expansion of Cyclopropanols and Cyclobutanols
}

Jordan P. Markham, Steven T. Staben, and F. Dean Toste

Department of Chemistry, University of California, Berkeley, California 94720

\section{Experimental}

General Information: Unless otherwise noted all commercial materials were used without further purification. Small-scale reactions $(<3 \mathrm{~mL})$ were carried out in Fisher Scientific disposable scintillation vials. Dichloromethane was obtained from EMD and used without further purification. Alkynyl cyclopropanols were prepared according to literature procedures. ${ }^{1}$ Flash column chromatography was carried out on Merck 60 silica gel $(32-63 \mu \mathrm{m}) .{ }^{1} \mathrm{H}$ and ${ }^{13} \mathrm{C}$ NMR spectra were recorded with Bruker AV-300, AVQ and AVB-400 and DRX-500 spectrometers and referenced to $\mathrm{CDCl}_{3}$ or $\mathrm{C}_{6} \mathrm{D}_{6}$. Mass spectral and $\mathrm{CHN}$ data were obtained via the Micro-Mass/Analytical Facility operated by the College of Chemistry, University of Berkeley.

\section{Representative procedure for ring expansion}

1-(3,3-dimethylbutynyl)cyclopropanol $(1.00 \mathrm{mmol})$ was added to a stirring suspension of gold triphenylphosphine chloride $(0.005 \mathrm{mmol})$ and silver hexafluoroantimonate $(0.005$ $\mathrm{mmol})$ in dichloromethane $(1 \mathrm{ml})$ and stirred for 6 hours at room temperature. After removal of the solvent under reduced pressure, silica gel flash chromatography eluting with $10 \%$ ethyl acetate in hexanes afforded cyclobutanone product as a white crystalline solid (157 mg, 99\%).

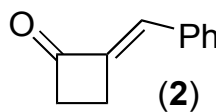

(2) (E)-2-Benzylidene-cyclobutanone (2): 12 hours with $0.5 \%$ catalyst. Yield: $157 \mathrm{mg}$ white crystalline solid, 95\% (silica gel chromatography, eluent $10 \%$ ethyl acetate in hexanes); ${ }^{1} \mathrm{H}$ NMR (400 MHz, $\left.\mathrm{CDCl}_{3}\right) \delta$ 7.54-7.51 (m, 2H), 7.44-7.39 (m, 3H), 7.04 (t, $J=2.8 \mathrm{~Hz}, 1 \mathrm{H}) 3.18-3.14(\mathrm{~m}, 2 \mathrm{H}), 3.02-2.99(\mathrm{~m}, 2 \mathrm{H}) ;{ }^{13} \mathrm{C}\left(125 \mathrm{MHz}, \mathrm{CDCl}_{3}\right) \delta 199.7$, 146.1, 134.5, 130.0, 128.9, 128.5, 126.4, 45.7, 23.5; IR: 2960, 1736, $1645 \mathrm{~cm}^{-1}$; HRMS (EI) calc'd for $\mathrm{C}_{11} \mathrm{H}_{10} \mathrm{O}$ : 158.0733, found: 158.0732; Anal. calc'd for $\mathrm{C}_{11} \mathrm{H}_{10} \mathrm{O}: \mathrm{C}, 83.51$; H, 6.37. Found: C, 83.50; H, 6.17. 


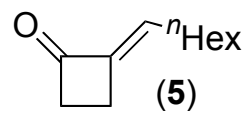

(E)-2-Heptylidene-cyclobutanone (5): 12 hours with 1\% catalyst. Yield: $94 \mathrm{mg}$ colorless oil, 81\% (silica gel chromatography, eluent 5\% ether in hexanes); ${ }^{1} \mathrm{H}$ NMR (500 MHz, $\left.\mathrm{C}_{6} \mathrm{D}_{6}\right) \delta$ 6.15-6.05 (m, 1H), 2.47 (dd, $\left.J=10.8,10.8 \mathrm{~Hz}, 2 \mathrm{H}\right), 2.09-2.01$ (m, 2H), $1.70(\mathrm{dd}, J=14.4,7.2 \mathrm{~Hz}, 2 \mathrm{H}), 1.25-1.03(\mathrm{~m}, 8 \mathrm{H}), 0.86\left(\mathrm{t}, J=7.2 \mathrm{~Hz}, 3 \mathrm{H} ;{ }^{13} \mathrm{C}\right.$ NMR (100 MHz, $\left.\mathrm{C}_{6} \mathrm{D}_{6}\right) \delta 197.0,148.9,129.5,43.6,31.9,29.2,28.8,28.4,22.9,20.2$, 14.3; HRMS (EI) calc'd for $\mathrm{C}_{11} \mathrm{H}_{18} \mathrm{O}$ : 166.1357, found: 166.1358 .

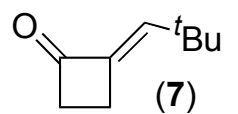

(7) (E)-2-(2,2-Dimethylpropylidene)-cyclobutanone (7): 6 hours with $0.5 \%$ catalyst. Yield: $136 \mathrm{mg}$ colorless oil, 98\% (silica gel chromatography, eluent 5\% ether in hexanes); ${ }^{1} \mathrm{H}$ NMR (400 MHz, $\left.\mathrm{CDCl}_{3}\right) \delta 6.17$ (t, $\left.J=2.8 \mathrm{~Hz}, 1 \mathrm{H}\right) 2.94-2.89$ (m, 2H), 2.772.70 (m, 2H), 1.07 (s, 9H); ${ }^{13} \mathrm{C}$ NMR (100 MHz, $\left.\mathrm{CDCl}_{3}\right) \delta$ 200.8, 144.1, 140.0, 45.0, 33.7, 29.1, 29.1, 22.1; IR: 2960, 2862, 1758, $1660 \mathrm{~cm}^{-1}$; HRMS (EI) calc'd for $\mathrm{C}_{9} \mathrm{H}_{14} \mathrm{O}$ : 138.1045, found: 138.1040; Anal. calc'd for $\mathrm{C}_{9} \mathrm{H}_{14} \mathrm{O}$ : C, 78.21; H, 10.21. Found: C, 78.14; H, 10.38 .

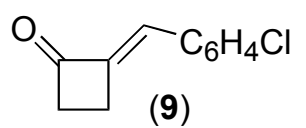

(E)-2-(4-Chlorobenzylidene)cyclobutanone (9): 12 hours with $0.5 \%$ catalyst. Yield: $45 \mathrm{mg}$ white crystalline solid, 93\% (silica gel chromatography, eluent $20 \%$ ethyl acetate in hexanes); ${ }^{1} \mathrm{H}$ NMR (400 MHz, $\left.\mathrm{CDCl}_{3}\right) \delta 7.43(\mathrm{~d}, J=8.4 \mathrm{~Hz}, 2 \mathrm{H})$, $7.36(\mathrm{~d}, J=8.4 \mathrm{~Hz}, 2 \mathrm{H}), 7.00$ (app. t, $J=2.6 \mathrm{~Hz}, 1 \mathrm{H}), 3.17-3.13$ (m, 2H), 2.98-2.93 (m, $2 \mathrm{H}) ;{ }^{13} \mathrm{C} \mathrm{NMR}\left(100 \mathrm{MHz}, \mathrm{CDCl}_{3}\right) \delta 199.2,146.6,135.9,133.0,131.1,129.2,125.0$, 45.8, 23.4; IR: 2975, 1733, $1648 \mathrm{~cm}^{-1}$; Anal. calc'd for $\mathrm{C}_{11} \mathrm{H}_{9} \mathrm{OCl}$ : C, 58.58; H, 4.71 . Found: C, 68.72; H, 4.94. HRMS (EI) calc'd for $\mathrm{C}_{11} \mathrm{H}_{9} \mathrm{OCl}$ : 192.0342, found: 192.0343 .

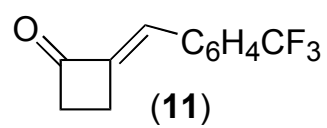

(E)-2-(4-(Trifluoromethyl)benzylidene)cyclobutanone (11): 12 hours with $0.5 \%$ catalyst. Yield: $108 \mathrm{mg}$ white crystalline solid, 96\% (silica gel chromatography, eluent $20 \%$ ethyl acetate in hexanes); ${ }^{1} \mathrm{H}$ NMR (400 $\left.\mathrm{MHz}, \mathrm{CDCl}_{3}\right) \delta$ $7.63(\mathrm{~d}, J=8.4 \mathrm{~Hz}, 2 \mathrm{H}), 7.58$ (d, $J=8.4 \mathrm{~Hz}, 2 \mathrm{H}), 7.01$ (bs, 1H) 3.20-3.16 (m, 2H), 3.03- 
$3.01(\mathrm{~m}, 2 \mathrm{H}) ;{ }^{13} \mathrm{C}\left(100 \mathrm{MHz}, \mathrm{CDCl}_{3}\right) \delta$ 199.1, 148.7, 138.0, 131.3, 130.0, 125.8, 124.5, 123.5, 46.1, 23.7; IR: 2934, 1730, $1650 \mathrm{~cm}^{-1}$; HRMS (EI) calc'd for $\mathrm{C}_{12} \mathrm{H}_{9} \mathrm{OF}_{4}$ : 226.0606, found: 226.0604. Anal. calc'd for $\mathrm{C}_{12} \mathrm{H}_{9} \mathrm{~F}_{3} \mathrm{O}$ : C, 63.72; H, 4.01. Found: C, 63.44; H, 4.03.

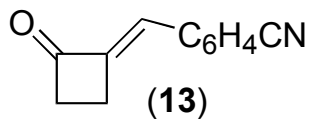

4-((E-(4-(Cyano)benzylidene)cyclobutanone (13): 12 hours with $0.5 \%$ catalyst. Yield: $91 \mathrm{mg}$ pale yellow solid, $98 \%$ (silica gel chromatography, eluent $20 \%$ ethyl acetate in hexanes); ${ }^{1} \mathrm{H} \mathrm{NMR}\left(400 \mathrm{MHz}, \mathrm{CDCl}_{3}\right) \delta 7.68(\mathrm{~d}, J=8.4 \mathrm{~Hz}, 2 \mathrm{H})$, 7.59 (d, $J=8.4 \mathrm{~Hz}, 2 \mathrm{H}), 7.00$ (t, $J=2.8 \mathrm{~Hz}, 1 \mathrm{H}) 3.29-3.19$ (m, $2 \mathrm{H}), 3.05-3.00(\mathrm{~m}, 2 \mathrm{H})$; IR: $2960,2224,1746,1642 \mathrm{~cm}^{-1} ;{ }^{13} \mathrm{C}\left(125 \mathrm{MHz}, \mathrm{CDCl}_{3}\right) \delta 198.8,149.6,138.9,132.5$, 130.1, 124.0, 118.3, 112.9, 46.2, 23.8. HRMS (EI) calc'd for $\mathrm{C}_{12} \mathrm{H}_{9} \mathrm{NO}$ : 183.0684, found: 183.0680 .

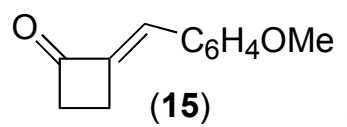

(E)-2-(4-Methoxybenzylidene)cyclobutanone (15): 12 hours with 0. 5\% catalyst. Yield: $88 \mathrm{mg}$ white crystalline solid, 94\% (silica gel chromatography, eluent $5 \%$ ethyl acetate in hexanes); ${ }^{1} \mathrm{H}$ NMR $\left(400 \mathrm{MHz}, \mathrm{CDCl}_{3}\right) \delta 7.46(\mathrm{~d}, J=8.8 \mathrm{~Hz}, 2 \mathrm{H})$, 6.99 (t, $J=2.8 \mathrm{~Hz}, 1 \mathrm{H}), 6.92$ (d, $J=8.8 \mathrm{~Hz}, 2 \mathrm{H}), 3.84(\mathrm{~s}, 3 \mathrm{H}), 3.13-3.09(\mathrm{~m}, 2 \mathrm{H}), 2.95-$ $2.90(\mathrm{~m}, 2 \mathrm{H}) ;{ }^{13} \mathrm{C}$ NMR $\left(100 \mathrm{MHz}, \mathrm{CDCl}_{3}\right) \delta 199.5,161.1,143.5,131.8,127.2,126.3$, 114.4, 55.3, 45.5, 23.2; IR: 2974, 1733, $1648 \mathrm{~cm}^{-1}$; HRMS (EI) calc'd for $\mathrm{C}_{12} \mathrm{H}_{12} \mathrm{O}_{2}$ : 188.0837, found: 188.0834. Anal. calc'd for $\mathrm{C}_{12} \mathrm{H}_{12} \mathrm{O}_{2}: \mathrm{C}, 76.57 ; \mathrm{H}, 6.43$. Found: $\mathrm{C}$, 76.69; H, 6.40 .

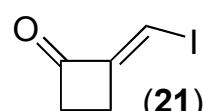

(21) (E)-2-(Iodomethylene)cyclobutanone (21) : Reaction with 1\% catalyst loading for $8 \mathrm{hrs}$ at $40^{\circ} \mathrm{C}$ gave $146 \mathrm{mg}(88 \%)$ of an off-white solid (silica-gel chromatography 10:1 hexanes: $\left.\mathrm{Et}_{2} \mathrm{O}\right) .{ }^{1} \mathrm{H} \mathrm{NMR}\left(\mathrm{CDCl}_{3}\right) \delta 7.44(\mathrm{t}, J=3.0,1 \mathrm{H}), 2.89(\mathrm{t}, J$ $=8.0,2 \mathrm{H}), 2.50(\mathrm{td}, J=8.0,3.0,2 \mathrm{H}) .{ }^{13} \mathrm{C} \mathrm{NMR}\left(\mathrm{CDCl}_{3}\right) \delta 192.41,160.71,85.33,40.58$, 26.14. IR: $3053,2930,1750,1622 \mathrm{~cm}^{-1}$. This compound decomposed rapidly at room temperature. Anal. Calcd.: C, 28.87; H, 2.42, found: C, 25.47; H, 2.02. 


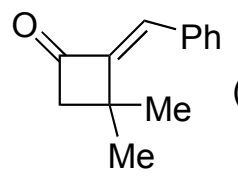

(23)

(E)-2-Benzylidene-3,3-dimethylcyclobutanone (23): 12 hours with 1\% catalyst. Yield: $61 \mathrm{mg}, 61 \%$ pale yellow oil (silica gel chromatography, eluent 2\% acetone in hexanes); ${ }^{1} \mathrm{H}$ NMR (400 $\left.\mathrm{MHz}, \mathrm{CDCl}_{3}\right) \delta$ 7.50-7.47 (m, 2H), 7.43-7.35 (m, 3H), $7.03(\mathrm{~s}, 1 \mathrm{H}), 2.92(\mathrm{~s}, 2 \mathrm{H}), 1.59(\mathrm{~s}, 6 \mathrm{H}) ;{ }^{13} \mathrm{C}\left(100 \mathrm{MHz}, \mathrm{CDCl}_{3}\right) \delta$ 200.2, 153.2, 133.4, 130.9, 129.7, 128.8, 127.2, 60.2, 38.0, 26.2; IR: 2959, 1742, $1632 \mathrm{~cm}^{-1}$; HRMS (EI) calc'd for $\mathrm{C}_{8} \mathrm{H}_{14} \mathrm{OSi}$ : 154.0809, found: 154.0814; Anal. calc'd for $\mathrm{C}_{8} \mathrm{H}_{14} \mathrm{OSi}$ : C, 62.281; H, 9.15. Found: C, 62.41; H, 9.21.

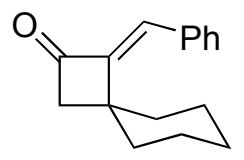

(25)

(E)-1-Benzylidenespiro[3.5]nona-2-one (25): Reaction with 5\% catalyst loading for $48 \mathrm{hr}$ at room temperature gave $63 \mathrm{mg}(74 \%)$ of a light yellow oil (4:1 mixture with a cyclopentenone isomer, silica gel chromatography 15:1 hexanes:EtOAc). ${ }^{1} \mathrm{H}$ NMR $\left(\mathrm{CDCl}_{3}\right): \delta 7.55(\mathrm{~m}, 2 \mathrm{H}), 7.45-7.35(\mathrm{~m}, 3 \mathrm{H}), 7.05(\mathrm{~s}, 1 \mathrm{H})$, $2.85(\mathrm{~s}, 2 \mathrm{H}), 2.08(\mathrm{~m}, 2 \mathrm{H}), 1.85-1.30(\mathrm{~m}, 8 \mathrm{H}) .{ }^{13} \mathrm{C} \mathrm{NMR}\left(\mathrm{CDCl}_{3}\right) \delta$ 200.57, 153.01, 134.61 130.99, 129.61, 128.71, 127.72, 56.05, 44.15, 34.06, 25.51, 23.43; IR: 2927, $2853,1742,1632 \mathrm{~cm}^{-1}$

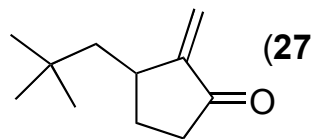

2-Methylene-3-neopentylcyclopentanone (27): 10 hours with 1\% catalyst. Yield: $16 \mathrm{mg}, 76 \%$ colorless oil (silica gel chromatography, eluent 5\% ethyl acetate in hexanes); ${ }^{1} \mathrm{H}$ NMR $\left(400 \mathrm{MHz}, \mathrm{CDCl}_{3}\right) \delta 6.03(\mathrm{~d}, \mathrm{~J}=3.2 \mathrm{~Hz}, 1 \mathrm{H}), 5.23(\mathrm{~d}, \mathrm{~J}=$ $2.8 \mathrm{~Hz}, 1 \mathrm{H}), 2.76-2.66$ (m, 1H), 2.46-2.21 (m, 3H), 1.69 (dd, J = 14.4, $3.2 \mathrm{~Hz}, 1 \mathrm{H}), 1.56-$ $1.45(\mathrm{~m}, 1 \mathrm{H}), 1.33(\mathrm{dd}, \mathrm{J}=14.0,8.4 \mathrm{~Hz}, 1 \mathrm{H}), 0.99(\mathrm{~s}, 9 \mathrm{H}) ;{ }^{13} \mathrm{C}\left(100 \mathrm{MHz}, \mathrm{CDCl}_{3}\right) \delta$ 207.3, 150.3, 116.3, 48.9, 38.3, 37.5, 31.0, 30.1, 29.7; HRMS (EI) calc'd for $\mathrm{C}_{11} \mathrm{H}_{18} \mathrm{O}$ : 166.1358, found: 166.1336 . 


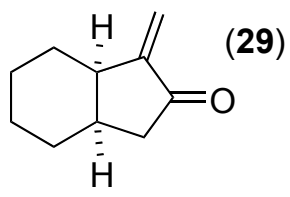

(3aR,7aR)-Hexahydro-1-methylene-1H-inden-2(3H)-one

(29):

Reaction with $2 \%$ catalyst loading for $24 \mathrm{hr}$ at room temperature gave $66 \mathrm{mg}(66 \%)$ of a colorless oil (silica gel chromatography 8:1 hexanes: $\mathrm{Et}_{2} \mathrm{O}$ ). 29 had characterization data consistent with that of the literature ${ }^{2}:{ }^{1} \mathrm{H}$ NMR $\left(\mathrm{CDCl}_{3}\right) \delta 6.07(\mathrm{~d}, J=3.0,1 \mathrm{H}), 5.20(\mathrm{~d}, J$ $=2.3,1 \mathrm{H}), 2.84(\mathrm{~m}, 1 \mathrm{H}), 2.37-2.23(\mathrm{~m}, 2 \mathrm{H}), 2.11(\mathrm{dd}, J=17.1,2.1,1 \mathrm{H}), 1.91(\mathrm{br} \mathrm{m}, 1 \mathrm{H})$, $1.70(\mathrm{~m}, 1 \mathrm{H}), 1.61-1.50(\mathrm{~m}, 2 \mathrm{H}), 1.40(\mathrm{~m}, 1 \mathrm{H}), 1.34-1.22(\mathrm{~m}, 2 \mathrm{H}), 1.00(\mathrm{~m}, 1 \mathrm{H}) \cdot{ }^{13} \mathrm{C}$ NMR $\left(\mathrm{CDCl}_{3}\right) \delta$ 207.57, 146.97, 116.17, 44.36, 14.07, 33.55, 28.74, 26.02, 23.98, 20.63. IR: 2931, 2857, 1705, $1643 \mathrm{~cm}^{-1}$

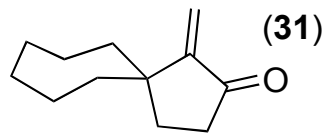

1-Methylenespiro[4.6] undeca-2-one (31): Reaction with 2\% catalyst loading for $20 \mathrm{hr}$ at $40^{\circ} \mathrm{C}$ gave $77 \mathrm{mg}$ (72\%) of a colorless oil (silica gel chromatography 15:1 hexanes:Et $2 \mathrm{O}) .{ }^{1} \mathrm{H}$ NMR $\left(\mathrm{CDCl}_{3}\right) \delta 5.92(\mathrm{br} \mathrm{s}, 1 \mathrm{H}), 5.24(\mathrm{br} \mathrm{s}, 1 \mathrm{H}), 2.32(\mathrm{t}, J=7.8$, $2 \mathrm{H}), 1.80(\mathrm{t}, J=7.8,2 \mathrm{H}), 1.65-1.47(\mathrm{~m}, 12 \mathrm{H}) .{ }^{13} \mathrm{C} \mathrm{NMR}\left(\mathrm{CDCl}_{3}\right) \delta 208.32,156.14$, 115.20, 46.44, 40.03, 35.34, 32.63, 29.71, 23.31. IR: 2922, 2853, 1723, $1630 \mathrm{~cm}^{-1}$ HRMS (EI) calc. for $\left[\mathrm{C}_{12} \mathrm{H}_{18} \mathrm{O}\right]^{+}$178.1358, found 178.1360. Anal. Calcd.: C, 80.85; H, 10.18, found: $\mathrm{C}, 80.83 ; \mathrm{H}, 10.36$.

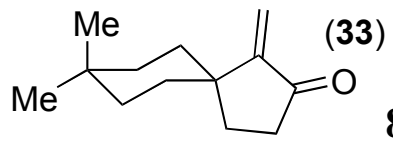

8,8-Dimethyl-1-methylenespiro[4.5]deca-2-one (33): Reaction with $2 \%$ catalyst loading for $16 \mathrm{hr}$ at $40^{\circ} \mathrm{C}$ gave $132 \mathrm{mg}(82 \%)$ as a light yellow oil (silica gel chromatography 10:1 hexanes: $\left.\mathrm{Et}_{2} \mathrm{O}\right) .{ }^{1} \mathrm{H}$ NMR $\left(\mathrm{CDCl}_{3}\right) \delta 6.02$ (br s, $\left.1 \mathrm{H}\right), 5.27$ (br s, $1 \mathrm{H}), 2.34(\mathrm{t}, J=7.9,2 \mathrm{H}), 1.86(\mathrm{t}, J=7.9,2 \mathrm{H}), 1.64(\mathrm{~m}, 2 \mathrm{H}), 1.46-1.30(\mathrm{~m}, 6 \mathrm{H}), 0.99$ (s, 3H), 0.98 (s, 3H). ${ }^{13} \mathrm{C}$ NMR $\left(\mathrm{CDCl}_{3}\right) \delta$ 208.29, 154.95, 115.98, 43.29, 35.35, 35.28, 33.09, 32.15, 29.55, 29.21, 24.76. IR: 2954, 2926, 2861, 1725, $1634 \mathrm{~cm}^{-1}$ Calcd.: C, 81.20; H, 10.48, found: C, 81.01; H,10.58. 


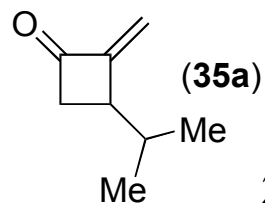

2-Methylene-3-isopropylcyclopentanone (35a): Reaction with 1\% catalyst loading for $10 \mathrm{~min}$ at room temperature gave $96 \mathrm{mg}(80 \%)$ of a light yellow oil (silica gel chromatography, 12:1 pentane:Et $\left.{ }_{2} \mathrm{O}\right) .{ }^{1} \mathrm{H} \mathrm{NMR}\left(\mathrm{CDCl}_{3}\right) \delta 5.75(\mathrm{~d}, J=2.7,1 \mathrm{H})$, $5.12(\mathrm{~d}, J=2.2,1 \mathrm{H}), 3.07(\mathrm{dd}, J=17.3,8.9,1 \mathrm{H}), 2.77(\mathrm{~m}, 1 \mathrm{H}), 2.67(\mathrm{dd}, J=17.3,6.0$, $1 \mathrm{H}), 1.82(\mathrm{~m}, 1 \mathrm{H}), 1.06(\mathrm{~d}, J=6.6,3 \mathrm{H}), 0.98(\mathrm{~d}, J=6.6,3 \mathrm{H}) .{ }^{13} \mathrm{C} \mathrm{NMR}\left(\mathrm{CDCl}_{3}\right) \delta$ 199.60, 157.65, 112.49, 48.61, 42.67, 32.11, 20.44, 20.13. IR: 2962, 2874, 1740, 1651 $\mathrm{cm}^{-1}$ HRMS (EI) calc. for $\left[\mathrm{C}_{8} \mathrm{H}_{12} \mathrm{O}\right]^{+} 124.0888$, found 124.0886 .

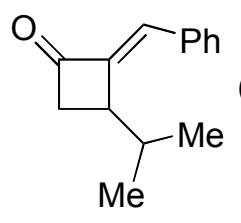

\section{$(35 b)$}

(E)-2-Benzylidene-3-isopropylcyclobutanone (35b): Reaction with $1 \%$ catalyst loading for $10 \mathrm{~min}$ at room temperature (determined by crude ${ }^{1} \mathrm{H} \mathrm{NMR}$ analysis to be a $4.6: 1$ (35b:36b) mixture of regioisomers) gave $28 \mathrm{mg}(82 \%)$ of a colorless oil (isolated as a 4:1 (35b:36b) mixture of regioisomers, silica gel chromatography 10:1 hexanes:Et $\left.{ }_{2} \mathrm{O}\right) .{ }^{1} \mathrm{H}$ NMR $\left(\mathrm{CDCl}_{3}\right) \delta 7.52(\mathrm{~m}, 2 \mathrm{H})$, 7.44-7.37 (m, $3 \mathrm{H}), 7.09(\mathrm{~d}, J=2.6,1 \mathrm{H}), 3.48(\mathrm{~m}, 1 \mathrm{H}), 3.08(\mathrm{dd}, J=17.5,9.0,1 \mathrm{H}), 2.87(\mathrm{dd}, J=17.5$, $5.1,1 \mathrm{H}), 2.41(\mathrm{~m}, 1 \mathrm{H}), 0.99(\mathrm{~d}, J=6.8,3 \mathrm{H}), 0.81(\mathrm{~d}, J=6.8,3 \mathrm{H}) .{ }^{13} \mathrm{C} \mathrm{NMR}\left(\mathrm{CDCl}_{3}\right) \delta$ $200.21,148.17,134.61,130.24,129.82,128.85,127.45,46.03,42.05,28.05,21.37$, 16.05. IR: 2959, 2871, 1744, $1638 \mathrm{~cm}^{-1}$ HRMS (EI) calc. for $\left[\mathrm{C}_{14} \mathrm{H}_{16} \mathrm{O}\right]^{+}$200.1201, found 200.1202 .

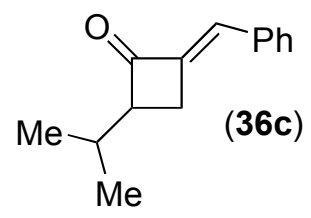

(E)-2-Benzylidene-4-isopropylcyclobutanone (36c): Reaction with $1 \%$ catalyst loading for $70 \mathrm{~min}$ at room temperature gave $51 \mathrm{mg}(91 \%)$ of a colorless solid (silica gel chromatography 10:1 hexanes:Et $2 \mathrm{O}) .{ }^{1} \mathrm{H} \mathrm{NMR}\left(\mathrm{CDCl}_{3}\right) \delta 7.54(\mathrm{~m}, 2 \mathrm{H})$, 7.42-7.36 (m, 3H), 7.04 (t, $J=2.5,1 \mathrm{H}), 3.16-3.05(\mathrm{~m}, 2 \mathrm{H}), 2.71$ (ddd, $J=15.5,5.5,2.5$, $1 \mathrm{H}), 2.00(\mathrm{~m}, 1 \mathrm{H}), 1.09(\mathrm{~d}, J=7.0,3 \mathrm{H}), 0.98(\mathrm{~d}, J=7.0,3 \mathrm{H}) .{ }^{13} \mathrm{C} \mathrm{NMR}\left(\mathrm{CDCl}_{3}\right) \delta$ $202.86,144.04,134.78,130.03,129.86,128.86,126.40,65.67,29.64,28.45,20.40$, 
20.24. IR: 2956, 2870, 1740, $1646 \mathrm{~cm}^{-1}$ HRMS (EI) calc. for $\left[\mathrm{C}_{14} \mathrm{H}_{16} \mathrm{O}\right]^{+}$200.1201, found 200.1204 .

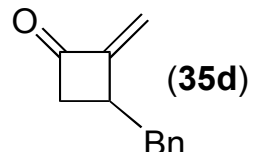

$\mathrm{Bn}$

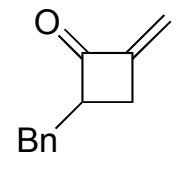

$(36 d)$

Methylene-4-benzylcyclobutanone (35d) and

Methylene-3-benzylcyclobutanone (36d): Reaction with 1\% catalyst loading for 5 min at room temperature (determined by crude ${ }^{1} \mathrm{H}$ NMR analysis to be a 1.7:1 (35d:36d) mixture of regioisomers) gave $44 \mathrm{mg}(88 \%)$ of a colorless oil (1.8:1 mixture of $\mathbf{3 5 d} \mathbf{3} \mathbf{3 6 d}$, silica gel chromatography 10:1 hexanes:Et $2 \mathrm{O}) .{ }^{1} \mathrm{H}$ NMR $\left(\mathrm{CDCl}_{3}\right)$ major isomer 35d: $\delta$ 7.35-7.28 (m, 2H), 7.26-7.18 (m, 3H), $5.72(\mathrm{~d}, J=2.6,1 \mathrm{H}), 4.87(\mathrm{~d}, J=1.9,1 \mathrm{H}), 3.32$ (m, 1H), $3.13(\mathrm{dd}, J=17.7,9.0,1 \mathrm{H}), 2.96(\mathrm{~m}, 1 \mathrm{H}), 2.80(\mathrm{~m}, 1 \mathrm{H}), 2.73(\mathrm{dd}, J=17.7,6.0$, $1 \mathrm{H})$ the following peaks could be observed for the minor isomer $36 \mathrm{~d}: \delta 5.78(\mathrm{t}, J=2.9$, $1 \mathrm{H}), 5.10(\mathrm{t}, J=2.3,1 \mathrm{H}), 3.53-3.46(\mathrm{~m}, 1 \mathrm{H}), 2.46-2.41(\mathrm{~m}, 1 \mathrm{H}) . \quad{ }^{13} \mathrm{C} \mathrm{NMR}\left(\mathrm{CDCl}_{3}\right)$ major isomer 35d: $\delta 199.14,158.12,139.43,128.75,128.69,126.45,112.59,50.71$, $40.21,36.26$ the following peaks could be observed for the minor isomer 36d: $\delta 202.25$, 152.72, 138.81, 126.36, 113.59, 57.91, 35.48, 28.9. HRMS (EI) calc. for $\left[\mathrm{C}_{12} \mathrm{H}_{12} \mathrm{O}\right]^{+}$ 172.0888, found 172.0888 .

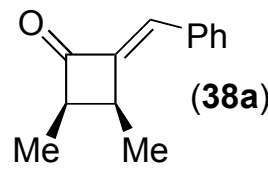

(E)-2-Benzylidene-cis-3,4-dimethylcyclobutanone (38a): 2 hours with 1\% catalyst. Yield: $861 \mathrm{mg}$ colorless oil, 99\% (silica gel chromatography, eluent 2\% ethyl acetate in hexanes); Structure confirmed by NOESY. ${ }^{1} \mathrm{H}$ NMR (500 $\left.\mathrm{MHz}, \mathrm{CDCl}_{3}\right) \delta$ 7.54-7.46 (m, 2H), 7.44-7.33 (m, 3H), 7.00 (d, J = 2.5 Hz, 1H), 3.60-3.49 (m, 1H), 3.49$3.42(\mathrm{~m}, 1 \mathrm{H}), 1.28(\mathrm{~d}, \mathrm{~J}=7.5 \mathrm{~Hz}, 3 \mathrm{H}), 1.16(\mathrm{~d}, \mathrm{~J}=7.5 \mathrm{~Hz}, 3 \mathrm{H}) ;{ }^{13} \mathrm{C}\left(125 \mathrm{MHz}, \mathrm{CDCl}_{3}\right) \delta$ 204.3, 149.8, 134.0, 130.2, 129.7, 128.8, 126.6, 55.6, 34.0, 12.0, 8.6; IR: 2962, 2853, 1745, $1643 \mathrm{~cm}^{-1}$; HRMS (EI) calc'd for $\mathrm{C}_{13} \mathrm{H}_{14} \mathrm{O}$ : 186.1045, found: 186.1045; Anal. calc'd for $\mathrm{C}_{13} \mathrm{H}_{14} \mathrm{O}$ : C, 83.83; H, 7.58. Found: C, 83.82; H, 7.70. 


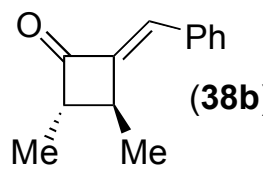

(E)-2-Benzylidene-trans-3,4-dimethylcyclobutanone (38a): 12 hours with 1\% catalyst. Yield: $70 \mathrm{mg}$ colorless oil, 94\% (silica gel chromatography, eluent 2\% ethyl acetate in hexanes); ${ }^{1} \mathrm{H}$ NMR (500 $\left.\mathrm{MHz} \mathrm{CDCl}_{3}\right) \delta$ 7.51-7.45 (m, 2H), 7.42-7.34 $(\mathrm{m}, 3 \mathrm{H}), 7.07(\mathrm{~d}, \mathrm{~J}=2.5 \mathrm{~Hz}, 1 \mathrm{H}), 3.06-2.98(\mathrm{~m}, 1 \mathrm{H}), 2.90-2.82(\mathrm{~m}, 1 \mathrm{H}), 1.41(\mathrm{~d}, \mathrm{~J}=7 \mathrm{~Hz}$, $3 \mathrm{H}), 1.27(\mathrm{~d}, \mathrm{~J}=7 \mathrm{~Hz}, 3 \mathrm{H}) ;{ }^{13} \mathrm{C}\left(125 \mathrm{MHz}, \mathrm{CDCl}_{3}\right) \delta$ 203.8, 148.8, 133.8, 130.3, 129.7, 128.7, 127.7, 61.0, 39.5, 17.2, 14.2; IR: 2961, 2868, 1739, $1640 \mathrm{~cm}^{-1}$; HRMS (EI) calc'd for $\mathrm{C}_{13} \mathrm{H}_{14} \mathrm{O}$ : 186.1045, found: 186.1045; Anal. calc'd for $\mathrm{C}_{13} \mathrm{H}_{14} \mathrm{O}$ : C, 83.83; H, 7.58. Found: C, 84.10; H, 7.71.

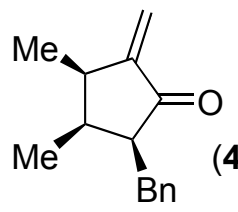

( \pm )-(2S,3S,4R)-2-Benzyl-3,4-dimethyl-5-methylenecyclopentanone (40): 12 hours with 1\% catalyst. Yield: $74 \mathrm{mg}$ colorless oil, 69\% (silica gel chromatography, eluent $2 \%$ ethyl acetate in hexanes); ${ }^{1} \mathrm{H}$ NMR $\left(500 \mathrm{MHz}, \mathrm{CDCl}_{3}\right) \delta$ 7.32-7.21 (m, 5H), $6.12(\mathrm{~d}, \mathrm{~J}=3.5 \mathrm{~Hz}, 1 \mathrm{H}), 5.23(\mathrm{~d}, \mathrm{~J}=2.5 \mathrm{~Hz}, 1 \mathrm{H}), 3.31(\mathrm{dd}, \mathrm{J}=10.0$, $4.5 \mathrm{~Hz}, 1 \mathrm{H}), 2.82-2.69$ (m, 2H), 2.54 (dd, 14.5, $10.0 \mathrm{~Hz}, 1 \mathrm{H}), 2.38-2.28$ (m, 1H), 1.15 (d, $7.0 \mathrm{~Hz}, 3 \mathrm{H}), 0.70(\mathrm{~d}, 7.5 \mathrm{~Hz}, 3 \mathrm{H}) ;{ }^{13} \mathrm{C}\left(125 \mathrm{MHz}, \mathrm{CDCl}_{3}\right) \delta 206.1,148.6,140.3,128.5$, 128.4, 126.0, 116.5, 56.2, 38.8, 35.0, 30.4, 13.5, 9.2; HRMS (EI) calc'd for $\mathrm{C}_{15} \mathrm{H}_{18} \mathrm{O}$ : 214.1358, found: 214.1357 .

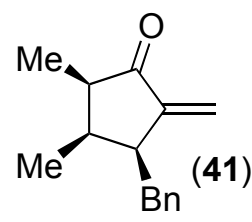

( \pm )-(3S,4R,5R)-3-Benzyl-4,5-dimethyl-2-methylenecyclopentanone

(41): Yield: $20 \mathrm{mg}$ colorless oil, 19\% (silica gel chromatography, eluent 2\% ethyl acetate in hexanes); ${ }^{1} \mathrm{H}$ NMR (500 MHz, $\left.\mathrm{CDCl}_{3}\right) \delta$ 7.35-7.19 (m, 5H), $6.12(\mathrm{~d}, \mathrm{~J}=3.0 \mathrm{~Hz}, 1 \mathrm{H})$, $5.26(\mathrm{~d}, \mathrm{~J}=3.0 \mathrm{~Hz}, 1 \mathrm{H}), 3.20-3.10(\mathrm{~m}, 2 \mathrm{H}), 2.68(\mathrm{dd}, \mathrm{J}=16.0,11.5 \mathrm{~Hz}, 1 \mathrm{H}), 2.47-2.38$ $(\mathrm{m}, 1 \mathrm{H}), 2.35-2.27(\mathrm{~m}, 1 \mathrm{H}), 1.06(\mathrm{~d}, \mathrm{~J}=7.0 \mathrm{~Hz}, 3 \mathrm{H}), 0.68(\mathrm{~d}, \mathrm{~J}=7.5 \mathrm{~Hz}, 3 \mathrm{H}) ;{ }^{13} \mathrm{C}(125$ $\left.\mathrm{MHz}, \mathrm{CDCl}_{3}\right) \delta$ 207.6, 147.2, 139.6, 128.5, 128.5, 126.2, 116.6, 48.8, 45.2, 34.8, 34.3, 9.4, 9.2; HRMS (EI) calc'd for $\mathrm{C}_{15} \mathrm{H}_{18} \mathrm{O}$ : 214.1358, found: 214.1359. 


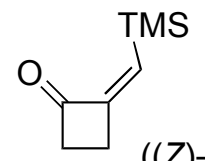

((Z)-44) (Z)-2-((Trimethylsilyl)methylene)cyclobutanone: Yield: $91 \mathrm{mg}, 83 \%$ colorless oil (silica gel chromatography, eluent $1 \%$ ethyl acetate in hexanes); ${ }^{1} \mathrm{H}$ NMR $\left(400 \mathrm{MHz}, \mathrm{CDCl}_{3}\right) \delta 5.91(\mathrm{t}, J=2.4 \mathrm{~Hz}, 1 \mathrm{H}), 2.87(\mathrm{dd}, J=8.4,8.4 \mathrm{~Hz}, 2 \mathrm{H}), 2.60$ (td, $J=$ 8.6, 2.1 Hz, 2H), 0.16 (s, 9H); ${ }^{13} \mathrm{C}\left(100 \mathrm{MHz}, \mathrm{CDCl}_{3}\right) \delta 200.3,162.0,138.0,42.8,24.3$, 1.1; HRMS (EI) calc'd for $\mathrm{C}_{8} \mathrm{H}_{14} \mathrm{OSi}$ : 154.0809, found: 154.0814; Anal. calc'd for $\mathrm{C}_{8} \mathrm{H}_{14} \mathrm{OSi}$ : C, 62.281; H, 9.15. Found: C, 62.41; H, 9.21.

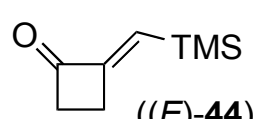

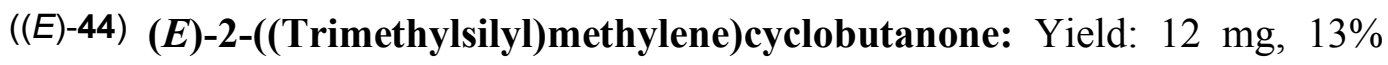
colorless oil (silica gel chromatography, eluent $1 \%$ ethyl acetate in hexanes); ${ }^{1} \mathrm{H}$ NMR $\left(400 \mathrm{MHz}, \mathrm{CDCl}_{3}\right) \delta 6.37$ (t, $\left.J=3.0 \mathrm{~Hz}, 1 \mathrm{H}\right), 2.99$ (dd, $\left.J=8.4,8.4 \mathrm{~Hz}, 2 \mathrm{H}\right), 2.71$ (td, $J=$ 8.6, $2.1 \mathrm{~Hz}, 2 \mathrm{H}), 0.17(\mathrm{~s}, 9 \mathrm{H}) ;{ }^{13} \mathrm{C}\left(100 \mathrm{MHz}, \mathrm{CDCl}_{3}\right) \delta 199.6,161.4,130.4,44.7,24.0$, 1.2; HRMS (EI) calc'd for $\mathrm{C}_{8} \mathrm{H}_{14} \mathrm{OSi}$ : 154.0809, found: 154.0814; Anal. calc'd for $\mathrm{C}_{8} \mathrm{H}_{14} \mathrm{OSi}$ : C, 62.281; H, 9.15. Found: C, 62.41; H, 9.21.

\section{References}

1) for example see: a) Salaün, J.; Bennani, F.; Compain, J.-C.; Fadel, A.; Oliver, J. J. Org. Chem. 1980, 45, 4129. b) Iwasawa, N.; Matsuo, T.; Iwamoto, M.; Ikeno, T. J. Am. Chem. Soc. 1998, 120, 3903. c) Trost, B. M.; Keeley, D. E.; Arndt, H. C.; Bogdanowicz, M. J. J. Am Chem. Soc. 1977, 99, 3088. d) Ghosez, L.; Montaigne, R.; Vanlierde, H.; Mollet, P. Tetrahedron, 1971, 27, 615.

2) Terakado, M.; Murai, K.; Miyazawa, M. Tetrahedron, 1994, 50, 5705. 